\title{
CORRECTION
}

\section{Correction to: Infection-related ventilator-associated complications in ICU patients colonised with extended-spectrum $\beta$-lactamase-producing Enterobacteriaceae}

\author{
François Barbier ${ }^{1}$, Sébastien Bailly² ${ }^{2}$ Carole Schwebel $^{3}$, Laurent Papazian ${ }^{4}$, Élie Azoulay ${ }^{5}$, Hatem Kallel ${ }^{6}$, \\ Shidasp Siami ${ }^{7}$, Laurent Argaud ${ }^{8}$, Guillaume Marcotte ${ }^{9}$, Benoît Misset ${ }^{10}$, Jean Reignier ${ }^{11}$, Michaël Darmon ${ }^{5}$, \\ Jean-Ralph Zaharr ${ }^{12}$, Dany Goldgran-Toledano ${ }^{13}$, Étienne de Montmollin ${ }^{14}$, Bertrand Souweine ${ }^{15}$, \\ Bruno Mourvillier ${ }^{16}$ and Jean-Francois Timsit ${ }^{2,16^{*}}$ for the OUTCOMEREA Study Group
}

๑ 2018 Springer-Verlag GmbH Germany, part of Springer Nature and ESICM

\section{Correction to: Intensive Care Med} https://doi.org/10.1007/s00134-018-5154-4

The members of the OUTCOMEREA Study Group were provided in such a way that they could not be indexed as collaborators on PubMed. The publisher apologizes for this error and is pleased to list the members of the group here.

\section{Members of the OUTCOMEREA Study Group-Sci- entific Committee \\ Jean-François Timsit (Medical and Infectious Diseases ICU, Bichat-Claude Bernard Hospital, Paris, France; UMR 1137 Inserm-Paris Diderot University IAME, F75018, Paris) \\ Elie Azoulay (Medical ICU, Saint Louis Hospital, Paris, France) \\ Maïté Garrouste-Orgeas (ICU, Saint-Joseph Hospital, Paris, France) \\ Jean-Ralph Zahar (Infection Control Unit, Angers Hos- pital, Angers, France) \\ Michael Darmon (Medical ICU, Saint Etienne Univer- sity Hospital, St Etienne, France)}

\footnotetext{
${ }^{*}$ Correspondence: jean-francois.timsit@aphp.fr

${ }^{16}$ Medical and Infectious Diseases ICU, Bichat-Claude Bernard Hospital,

APHP, 46 Rue Henri-Huchard, 75877 Paris Cedex 18, France

Full author information is available at the end of the article
}

The original article can be found online at https://doi.org/10.1007/s00134018-5154-4.

\section{6

Christophe Clec'h (ICU, Avicenne Hospital, Bobigny, and UMR 1137 Inserm, Paris Diderot University IAME, F75018, Paris, France).

Biostatistical and information system expertise: Jean-Francois Timsit (Medical and Infectious Diseases ICU, Bichat-Claude Bernard Hospital, Paris, France; UMR 1137 Inserm -Paris Diderot University IAME, F75018, Paris)

Corinne Alberti (Medical Computer Sciences and Biostatistics Department, Robert Debré Hospital, Paris, France)

Adrien Français (Integrated Research Center U823, Grenoble, France)

Aurélien Vesin (OUTCOMEREA organization and Integrated Research Center U823, Grenoble, France)

Stephane Ruckly (OUTCOMEREA organization and Inserm UMR 1137 IAME, F75018, Paris)

Sébastien Bailly (Grenoble University Hospital Inserm UMR 1137 IAME, F75018, Paris)

Christophe Clec'h (ICU, Avicenne Hospital, Bobigny, and Inserm UMR 1137 IAME, F75018, Paris, France)

Frederik Lecorre (Supelec, France)

Didier Nakache (Conservatoire National des Arts et Métiers, Paris, France)

Aurélien Vannieuwenhuyze (Tourcoing, France). 


\section{Investigators of the OUTCOMEREA database}

Dr Romain HERNU, Christophe Adrie (ICU, CH Melun, and Physiology, Cochin Hospital, Paris, France)

Carole Agasse (Medical ICU, University Hospital Nantes, France)

Bernard Allaouchiche (ICU, Pierre Benite Hospital, Lyon, France)

Olivier Andremont (ICU, Bichat Hospital, Paris, France)

Pascal Andreu (CHU Dijon, Dijon, France)

Laurent Argaud (Medical ICU, Hospices Civils de Lyon, Lyon, France)

Claire Ara-Somohano (Medical ICU, University Hospital, Grenoble, France)

Elie Azoulay (Medical ICU, Saint Louis Hospital, Paris, France)

François Barbier (Medico-surgical ICU, Orleans, France)

Déborah Boyer (ICU, CHU Rouen, France)

Jean-Pierre Bedos (ICU, Versailles Hospital, Versailles, France)

Thomas Baudry (Medial ICU, Edouard Heriot Hospital, Lyon, France)

Jérome Bedel (ICU, Versailles Hospital, Versailles, France)

Julien Bohé (ICU, Hôpital Pierre Benite, Lyon, France)

Lila Bouadma (ICU, Bichat Hospital, Paris, France)

Jeremy Bourenne (Réanimation des urgencies,

Timone-2; APHM, Marseille, France)

Noel Brule (Medical ICU, University Hospital Nantes,

France)

Cédric Brétonnière (Medical ICU, University Hospital

Nantes, France)

Christine Cheval (ICU, Hyeres Hospital, Hyeres,

France)

Julien Carvelli (Réanimation des urgencies, Timone-2;

APHM, Marseille, France)

Christophe Clec'h (ICU, Avicenne Hospital, Bobigny,

France)

Elisabeth Coupez (ICU, G Montpied Hospital, Clermont-Ferrand, France)

Martin Cour Medial ICU, Edouard Heriot hospital, Lyon, France)

Michael Darmon (ICU, Saint Etienne Hospital, Saint

Etienne, France)

Etienne de Montmollin (ICU, Delafontaine Hospital,

Saint Denis)

Loa Dopeux (ICU, G Montpied Hospital, ClermontFerrand, France)

Anne-Sylvie Dumenil (Antoine Béclère Hospital, Clamart, France)

Claire Dupuis (Bichat Hospital and UMR 1137 InsermParis Diderot University IAME, F75018, Paris, France)
Jean-Marc Forel (AP HM, Medical ICU, Hôpital Nord

Marseille)

Marc Gainnier (Réanimation des urgencies, Timone-2;

APHM, Marseille, France)

Charlotte Garret (Medical ICU, University Hospital

Nantes, France)

Steven Grangé (ICU, CHU Rouen, France)

Antoine Gros (ICU, Versailles Hospital, Versailles, France)

Akim Haouache (Surgical ICU, H Mondor Hospital, Creteil, France)

Romain Hernu (Medical ICU, Hospices Civils de Lyon,

Lyon, France)

Tarik Hissem (ICU, Eaubonne, France)

Vivien Hon Tua Ha (ICU, CH Meaux, France)

Sébastien Jochmans (ICU, CH Melun)

Jean-Baptiste Joffredo (ICU, G Montpied Hospital, Clermont-Ferrand, France)

Hatem Kallel (ICU, Cayenne General Hospital, Cayenne, France)

Guillaume Lacave (ICU, Versailles Hospital, Versailles, France)

Alexandre Lautrette (ICU, G Montpied Hospital, Clermont-Ferrand, France)

Virgine Lemiale (Medical ICU, Saint Louis Hospital,

Paris, France)

Mathilde Lermuzeaux (ICU, Bichat Hospital, Paris, France)

Guillaume Marcotte (Surgical ICU, Hospices Civils de

Lyon, Lyon, France)

Jordane Lebut (ICU, Bichat Hospital, Paris, France)

Maxime Lugosi (Medical ICU, University Hospital Grenoble, Grenoble, France)

Eric Magalhaes (ICU, Bichat Hospital, Paris, France)

Sibylle Merceron (ICU, Versailles Hospital, Versailles, France)

Bruno Mourvillier (ICU, Bichat Hospital, Paris, France)

Benoît Misset (ICU, Saint-Joseph Hospital, Paris, France and Medical ICU CHU Rouen, France)

Bruno Mourvillier (ICU, Bichat Hospital, Paris, France) Mathild Neuville (ICU, Bichat Hospital, Paris, France)

Laurent Nicolet (Medical ICU, University Hospital Nantes, France)

Johanna Oziel (Medico-surgical ICU, hôpital Avicenne APHP, Bobigny, France)

Laurent Papazian (Hopital Nord, Marseille, France)

Benjamin Planquette (Pulmonology ICU, George Pompidou Hospital, Paris, France)

Jean-Pierre Quenot (CHU Dijon, Dijon, France)

Aguila Radjou (ICU, Bichat Hospital, Paris, France)

Marie Simon (Medial ICU, Edouard Heriot Hospital, Lyon, France) 
Romain Sonneville (ICU, Bichat Hospital, Paris, France)

Jean Reignier (Medical ICU, University Hospital Nantes, France)

Bertrand Souweine (ICU, G Montpied Hospital, Clermont-Ferrand, France)

Carole Schwebel (ICU, A Michallon Hospital, Grenoble, France)

Shidasp Siami (ICU, Eaubonne, France)

Roland Smonig (ICU, Bichat Hospital, Paris, France)

Gilles Troché (ICU, Antoine Béclère Hospital, Clamart, France)

Marie Thuong (ICU, Delafontaine Hospital, Saint Denis, France)

Guillaume Thierry (ICU, Saint-Louis Hospital, Paris, France)

Dany Toledano (ICU, Gonesse Hospital, Gonesse, France)

Guillaume Van Der Meersch, Medical Surgical ICU, University Hospital Avicenne)

Marion Venot (Medical ICU, Saint Louis Hospital, Paris, France)

Olivier Zambon (Medical ICU, University Hospital Nantes, France)

\section{Study Monitors}

Julien Fournier, Caroline Tournegros, Stéphanie Bagur, Mireille Adda, Vanessa Vindrieux, Sylvie de la Salle, Pauline Enguerrand, Loic Ferrand, Vincent Gobert,
Stéphane Guessens, Helene Merle, Nadira Kaddour, Boris Berthe, Samir Bekkhouche, Kaouttar Mellouk, Mélaine Lebrazic, Carole Ouisse, Diane Maugars, Christelle Aparicio, Igor Theodose, Manal Nouacer, Veronique Deiler, Myriam Moussa, Atika Mouaci, Nassima Viguier and Sophie Letrou.

\begin{abstract}
Author details
${ }_{1}^{1}$ Medical ICU, La Source Hospital, CHR Orléans, Orléans, France. ${ }^{2}$ UMR 1137, IAME Team 5, DeSCID: Decision SCiences in Infectious Diseases, Control and Care, INSERM, Paris Diderot, Sorbonne Paris Cité University, Paris, France. ${ }^{3}$ Medical ICU, Albert Michallon University Hospital, Grenoble, France. ${ }^{4}$ Respiratory and Infectious Diseases ICU, North Hospital, Marseille, France. ${ }^{5}$ Medical ICU, Saint-Louis Hospital, APHP, Paris, France. ${ }^{6}$ Medical Surgical ICU, Andrée Rosemon Hospital, Cayenne, France. ${ }^{7}$ Medical Surgical ICU, Corbeil-Essone Hospital, Corbeil-Essone, France. ${ }^{8}$ Medical ICU, Edouard-Herriot University Hospital, Lyon, France. ${ }^{9}$ Surgical ICU, Edouard-Herriot University Hospital, Lyon, France. ${ }^{10}$ Medical ICU, Charles Nicolle University Hospital, Rouen, France. ${ }^{11}$ Medical ICU, Hôtel-Dieu University Hospital, Nantes, France. ${ }^{12}$ Infection Control Unit, Avicenne Hospital, Bobigny, France. ${ }^{13}$ Medical-Surgical ICU, General Hospital, Montfermeil, France. ${ }^{14}$ Medical-Surgical ICU, Delafontaine Hospital, Saint-Denis, France. ${ }^{15}$ Medical ICU, Gabriel Montpied Hospital, Clermont-Ferrand, France. ${ }^{16}$ Medical and Infectious Diseases ICU, Bichat-Claude Bernard Hospital, APHP, 46 Rue Henri-Huchard, 75877 Paris Cedex 18, France.
\end{abstract}

Published online: 15 June 2018 